\title{
Changes in poleward atmospheric energy transport over a wide range of climates: Energetic and diffusive perspectives and a priori theories
}

\author{
Timothy M. MerLis* \\ McGill University, Montréal, QC, Canada \\ NiCOLE FeldL \\ University of California, Santa Cruz, CA, USA \\ Rodrigo Caballero \\ Stockholm University, Stockholm, Sweden
}

\begin{abstract}
The midlatitude poleward atmospheric energy transport increases in radiatively forced simulations of warmed climates across a range of models from comprehensive coupled general circulation models (GCMs) to idealized aquaplanet moist GCMs to diffusive moist energy balance models. These increases have been rationalized from two perspectives. The energetic (or radiative) perspective takes the atmospheric energy budget and decomposes energy flux changes (radiative forcing, feedbacks, or surface fluxes) to determine the energy transport changes required by the budget. The diffusive perspective takes the net effect of atmospheric macroturbulence to be a diffusive energy transport down-gradient, so a change in transport can arise from changes in mean energy gradients or turbulent diffusivity. Here, we compare these perspectives in idealized moist, gray-radiation GCM simulations over a wide range of climate states. The energetic perspective has a dominant role for radiative forcing in this GCM, with cancellation between the components of the temperature feedback that can account for the GCM's non-monotonic energy transport changes. Comprehensive CMIP5 GCM simulations have similarities in the northern hemisphere to the idealized GCM, though a comprehensive GCM over several $\mathrm{CO}_{2}$ doublings has a distinctly different feedback structure evolution. The diffusive perspective requires a non-constant diffusivity to account for the idealized GCM-simulated changes, with important roles for the eddy velocity, dry static stability, and horizontal energy gradients. Beyond diagnostic analysis, GCM-independent a priori theories for components of the temperature feedback are presented that account for changes without knowledge of a perturbed climate state, suggesting that this is the more parsimonious perspective.
\end{abstract}

\section{Introduction}

The poleward atmospheric and oceanic energy transports play a fundamental role in determining the climatological equator-to-pole surface temperature contrast, cooling the tropics and warming polar latitudes. Therefore, changes in these energy transports are a critical aspect of climate change projections, with implications for tropical heat stress, sea ice loss, and a host of other climate impacts. These energy transport changes are projected using global climate models (GCMs) that explicitly simulate large-scale flows in the atmosphere and ocean with parameterizations for unresolved flows. Examining different perspectives for how energy transports change with climate can elucidate robust aspects of future climate projections and may offer insight into the origin of spread between climate models. Here, we consider a wide range of climate states both because climates much colder or warmer than modern Earth

\footnotetext{
*Corresponding author: Timothy M. Merlis, timothy.merlis@mcgill.ca
}

are interesting in and of themselves and as a means of probing the limits of different perspectives.

Comprehensive GCMs typically have an increase in poleward energy transport as the climate warms, with some offsetting changes between atmosphere and ocean (Held and Soden 2006; Hwang et al. 2011b). In idealized aquaplanet atmospheric GCM simulations, there are also increases in poleward transport about Earth-like climate states, though there can be more subtle changes when a wide range of climates is simulated (O'Gorman and Schneider 2008b). Transports in comprehensive atmospheric GCM simulations performed over a wide range of climates have been shown to depend on the globalmean temperature and meridional temperature gradients, in line with diffusive expectations (Caballero and Langen 2005). Finally, diffusive moist energy balance models (EBMs) have increased energy transport with warming and are amenable to thorough analysis (Flannery 1983; Rose et al. 2014; Merlis and Henry 2018).

There is an extensive literature treating, justifying, and refining formulations of the atmospheric macrotur- 
bulence's energy transport as downgradient diffusion (e.g., Sellers 1969; North 1975; Flannery 1983; Stone and Yao 1990; Schneider and Walker 2008; Bischoff and Schneider 2014). One challenge is that mean energy gradients depend on eddy transports - our starting climatic motivation. Also, the diffusivity depends on eddy length and eddy velocity, which could, in turn, be related back to mean field quantities (e.g., via baroclinic instability theory or mean available potential energy). Nevertheless, it also appears that simplistic formulations that neglect climate dependence of diffusivity can be adequate for climate changes relevant to the next century (e.g., Armour et al. 2019).

The energy budget perspective is an appealing approach in that it is a textbook starting point for discussing the climatological northward energy transport (e.g., Hartmann 1994). It can be used for climate changes by relating top-of-atmosphere (TOA) energy budget changes to the spatial structure in radiative feedbacks (Zelinka and Hartmann 2012) and radiative forcing (Huang and Zhang 2014). GCM simulations have been used to reveal the importance of the spatial structure of the radiative forcing by flattening it via inhomogeneous perturbations to the carbon dioxide $\left(\mathrm{CO}_{2}\right)$ concentration, thereby eliminating the simulated energy transport increase from the standard uniformly perturbed $\mathrm{CO}_{2}$ concentration (Huang et al. 2016). A challenging dimension of this perspective is that knowledge of the spatial distribution of the feedbacks is required and depends in detail on the vertical and horizontal structure of climate-response fields (e.g., temperature, water vapor, cloud, and surface albedo). We note that neither local radiative feedbacks should be considered independent of the energy transport, nor should energy transport changes be considered independent of radiative feedback changes (Hwang et al. 2011a; Merlis 2014; Trossman et al. 2016; Feldl et al. 2017)

The energy budget perspective has been fruitfully applied to comprehensive GCM simulations (recent generations of the Coupled Model Intercomparison Project, CMIP, Zelinka and Hartmann 2012), while diffusive closures are typically developed or used in idealized GCM simulations (e.g., Bischoff and Schneider 2014) or comprehensive atmospheric GCM simulations with idealized boundary conditions (e.g., Caballero and Langen 2005; Caballero and Hanley 2012). Here, we use the TOA forcingfeedback decomposition of the TOA net radiation in an idealized GCM and compare the results to a diffusive closure previously described for the same GCM, as well as a simpler, widely used scaling. The simulations are perturbed by altering the optical depth in a gray radiation scheme to simulate a wide range of climate states. This GCM and this perturbation has been the subject of a range of climate studies (O'Gorman and Schneider 2008b; Merlis and Schneider 2011; Bischoff and Schneider 2014) and can be considered one of the central models in the hierarchy of climate models (Jeevanjee et al. 2017; Maher et al. 2019).
In addition, we compare the idealized GCM to comprehensive GCM simulations.

Armour et al. (2019) presented a closely related comparison of different perspectives on energy transport for CMIP5 simulations. They included an additional perspective that they called "dynamic" that amounts to diagnosing simulated energy transports with the classic Reynold's average flow decomposition into zonal-mean flow, transient eddy and stationary eddy components. Our focus on the mid-latitudes in aquaplanet simulations, where transient eddies dominate, justifies our choice to set aside this perspective.

Section 2 describes the GCMs that we analyze. Section 3 provides an overview of the energetic and diffusive closures in general and how they are evaluated for the idealized GCM. In section 4, we assess the energetic perspective in the idealized GCM simulations, put forth a priori theories that do not rely on knowledge of perturbed climate states ("GCM independent"), and compare idealized GCM results to comprehensive GCMs. The diffusive perspective is assessed in the idealized GCM in section 5 and avenues of GCM-independent theories are discussed. We conclude in section 6 .

\section{General Circulation Models \\ a. Idealized gray-radiation model description}

The simulations we present follow O'Gorman and Schneider (2008b), who in turn built on Frierson et al. (2006). There is an aquaplanet (zonally symmetric, water covered) lower boundary condition with a slab ocean ( $1 \mathrm{~m}$ depth) to allow the surface temperature to come into equilibrium with the radiative and turbulent surface fluxes. The atmosphere is idealized relative to comprehensive atmospheric GCMs in that the radiative transfer is a gray scheme. This eliminates radiative feedbacks of clouds and water vapor. Water vapor is introduced into the atmosphere via evaporative fluxes and is lost to condensation (via a super-saturation condensation scheme for resolved flows) and a simple moist convection scheme (Frierson 2007).

The model's gray radiation scheme has a prescribed (vertically and meridionally varying) optical depth. These simulations are the same as those of O'Gorman and Schneider (2008b), with additional simulations and diagnostics to perform the forcing and feedback analysis (described below and following, Henry and Merlis 2019). The simulations have T42 resolution and 30 unevenly-spaced $\sigma=p / p_{s}$ levels; they are integrated with a $600 \mathrm{~s}$ time-step for 3000 days, and averages over the final 2400 days are shown.

The climate is varied by changing the longwave optical depth by a multiplicative factor to generate climates with global-mean surface temperatures that span the range of $\approx 260$ to $315 \mathrm{~K}$. The global-mean column water vapor varies by more than a factor of 20 , following closely the 
Clausius-Clapeyron relation, and the global-mean precipitation varies by a factor of $\approx 6$ (O'Gorman and Schneider 2008b).

\section{b. Comprehensive climate models}

\section{1) CMIP5}

To investigate how the results of the gray radiation GCM compare to comprehensive GCM simulations for climate changes of the magnitude projected over the next century, we compare the radiative feedbacks and temperature change pattern to those of CMIP5 simulations. The feedbacks shown here use the conventional feedback decomposition (lapse rate, Planck, water vapor, surface albedo, cloud) with details in Feldl et al. (2020); they are computed using the radiative kernel technique (Soden et al. 2008) with the kernel of Pendergrass et al. (2018). The perturbation fields are the difference between the abrupt $4 \mathrm{xCO}_{2}$ and piControl CMIP5 experiments. Here, we focus on the temperature feedback components, as this is a relevant comparison to the idealized, gray radiation GCM results, but we note that there are important differences in local feedbacks from water vapor and shortwave changes (e.g., Zelinka and Hartmann 2012; Huang and Zhang 2014).

\section{2) CCSM3}

To assess how the results of the gray radiation GCM compare to a comprehensive GCM over a wide range of climates, we show the radiative feedbacks and temperature change pattern in simulations using the Community Climate System Model version 3 (CCSM3) forced with a broad range of elevated $\mathrm{CO}_{2}$ concentrations. CCSM3 includes a comprehensive atmospheric GCM, with cloud, surface albedo, water vapor, and temperature feedbacks. Caballero and Huber (2013) investigated the state dependence of this model's climate sensitivity over a range from $1 \times$ to $32 \times$ pre-industrial $\mathrm{CO}_{2}$ concentration (5 doublings) using the partial radiative perturbation method, and showed that global-mean cloud and water vapor feedbacks became more destabilizing in hot climates. That study employed Eocene boundary conditions and changing ocean heat transport. Here, we present results from an similar set of simulations spanning 6 doublings of $\mathrm{CO}_{2}$ using presentday continents with an unchanging prescribed ocean heat transport based on today's climate, for consistency with the idealized GCM. The feedback analysis method is exactly as described in Caballero and Huber (2013). Our interest here is in the evolution with climate state of the spatial structure of the radiative feedbacks and temperature change pattern, which can account for energy transports, as a point of comparison to the idealized, gray radiation GCM.

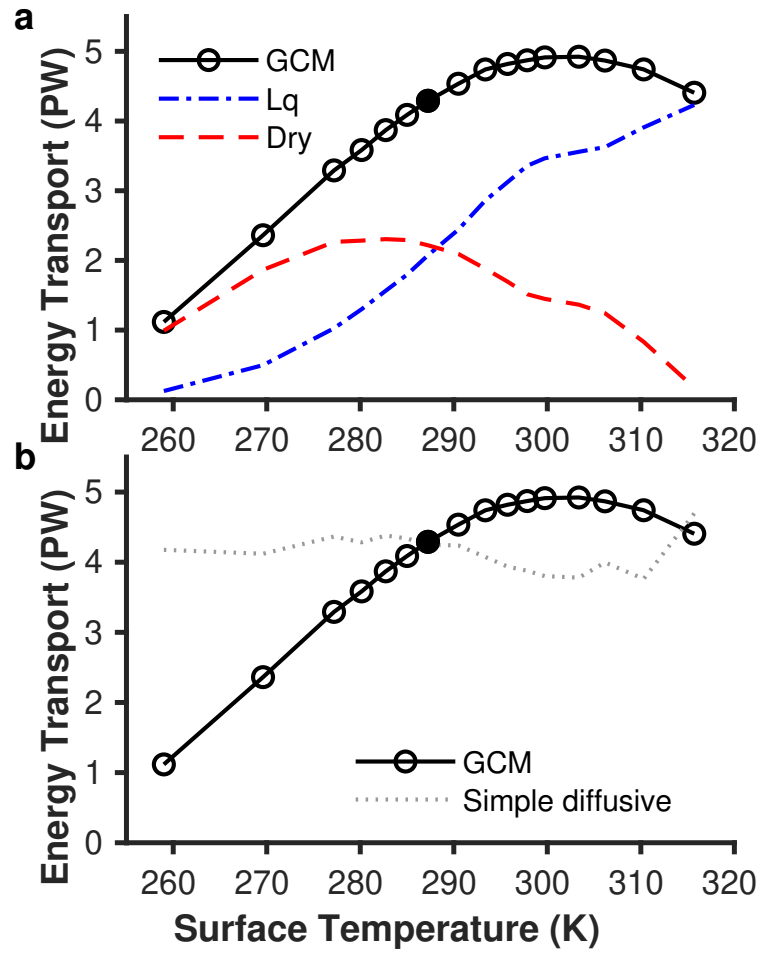

FIG. 1. (a) Vertically integrated northward energy transport at $50^{\circ} \mathrm{N}$ for GCM simulations (black) indicated by their global-mean surface temperature, with (blue) latent energy and (red) dry static energy transport components. (b) Evaluation of the simple diffusive closure (5) at $50^{\circ} \mathrm{N}$ using the mean moist static energy gradients at the lowest atmospheric level.

\section{c. A wide range of idealized GCM climates}

Figure 1a shows that the midlatitude energy transport, evaluated at $50^{\circ} \mathrm{N}$, varies non-monotonically with climate, increasing from cold climates to a maximum in the climate with a global-mean temperature near $300 \mathrm{~K}$ and decreasing with subsequent warming. In the climate states below that temperature, the rate of the increase per unit warming varies, with larger magnitude increases per unit warming in the cold climates than in those with Earth-like temperatures (i.e., a steeper slope on the left side of Fig. 1a than near the middle).

Some insight into the behavior can be found by decomposing the energy transport into latent $L_{v} q$ and dry static energy $c_{p} T+g z$ components (blue and red lines in Fig. 1a). The latent component of the energy transport monotonically increases with warming, albeit at a slower rate in hot climates. The dry static energy component of the energy transport has a maximum near the reference climate, decreasing toward both colder and warmer climates. We return to this in section 5 .

Figure 2a shows the meridional structure of the changes in energy transport between a climate with $6.1 \mathrm{~K}$ of global 
warming (second warmer than control in Fig. 1) and the control simulation (black line). The $0.45 \mathrm{PW}$ increase at $50^{\circ}$ is a $1.7 \%$ per $\mathrm{K}$ increase from the control. This result is broadly similar to the increases in radiatively forced warming scenarios in comprehensive GCMs, although there can be more detailed regional structure near regions of ice loss (Huang and Zhang 2014).

\section{Energetic and Diffusive Perspectives}

\section{a. Energy Budget}

\section{1) General Form}

The net radiation at the top of atmosphere $N_{T O A}$ is balanced by the vertically integrated divergence of the horizontal energy transport $\mathbf{F}$ :

$$
N_{T O A}=\nabla \cdot\{\mathbf{F}\}, \quad \mathbf{F}=\mathbf{F}_{\mathbf{a}}+\mathbf{F}_{\mathbf{o}},
$$

where the total energy transport $\mathbf{F}$ is comprised of the sum of the atmospheric $\mathbf{F}_{\mathbf{a}}$ and oceanic $\mathbf{F}_{\mathbf{o}}$ components. Here, we consider equilibrated slab ocean simulations without a representation of ocean energy transport, so $N_{T O A}=$ $\nabla \cdot\left\{\mathbf{F}_{\mathbf{a}}\right\}$.

The net radiation can be decomposed using the forcingfeedback analysis technique, a first-order Taylor series expansion in the surface temperature change (e.g., Feldl and Roe 2013):

$$
\Delta N_{T O A}=\mathcal{F}+\lambda \Delta T,
$$

with radiative forcing $\mathcal{F}$, climate feedback parameter $\lambda$, and surface temperature change $\Delta T$.

The feedback parameter can be further decomposed into individual feedbacks. The change in the TOA radiation associated with each of these terms can then be converted into a meridional energy transport:

$$
\Delta \mathbf{F}_{i}=2 \pi a^{2} \int_{-90^{\circ}}^{\phi}\left(\Delta \widetilde{N}_{T O A}\right)_{i} \cos (\phi) d \phi,
$$

where $a$ is planet radius, $\phi$ is latitude, index $(\cdot)_{i}$ indicates an individual feedback or forcing, $\widetilde{(\cdot)}$ is a departure from the global mean $\langle\cdot\rangle$.

To build expectations for this perspective, it is helpful to consider a forcing (or an individual feedback's effect on $N_{T O A}$ ) that is larger in the tropics than in the midlatitudes. This is a spatial structure that augments the climatological equator-to-pole decrease in net radiation and will therefore require an increase in poleward energy transport in an equilibrated climate state. Likewise, a destabilizing feedback process that is limited to high latitudes (e.g., ice albedo feedback) locally increases the radiative energy input and therefore less advective energy input is then needed at these latitudes, tending to weaken the convergence of the energy transport.

\section{2) Assessment in Idealized GCM}

The radiative forcing of the longwave optical depth change is computed as the difference between two simulations with the same prescribed climatological surface temperature, one with perturbed optical depth relative to the control. This is a troposphere-adjusted forcing. We calculate the radiative feedbacks as in Henry and Merlis (2019). This is a "local" feedback calculation (e.g., Feldl and Roe 2013), where the Planck feedback is a determined online by an additional call to the radiative transfer with the temperature perturbed by $1 \mathrm{~K}$ and the lapse rate feedback is determined as the residual. Therefore, the lapse rate feedback potentially includes second-order (nonlinear) terms in $\Delta T$. We have also used the radiative kernel method (Soden et al. 2008) to compute the lapse rate feedback and found similar results.

The wide range of climates that we examine somewhat complicates the feedback analysis approach, which is based on linearizing radiative fluxes about a reference climate state (e.g., Roe 2009). If the perturbations are all relative to the Earth-like reference, this leads to departures from linearity as the amplitude of the climate change gets large. If the perturbations are relative to different control climate states, this ensures the linearization is accurate, but requires additional information: the forcing and feedbacks are then based on detailed, time-dependent calculations from each of the "control" climate states. For example, multiplying the Planck feedback parameter computed from the Earth-like reference climate by various time-mean temperature changes from the wide range of climates requires less information than computing the Planck feedback parameter for each climate state, which uses double calls to the radiation module and time-varying temperature fields as input. In practice, we find that the results of interest do not sensitively depend on this choice, likely resulting from the simplicity of the radiation scheme and absence of additional feedbacks. The results we show vary the reference climate about which the perturbations are defined, for consistency with the feedback analysis of the CCSM3 simulations.

\section{b. Diffusive Closures}

In simplest form, diffusive closures represent the transport of the relevant energy (moist static energy $h$ for moist atmospheres) in terms of the time- and zonal-mean, indicated by $\overline{(\cdot)}$, energy gradient and a diffusivity $\mathcal{D}$ :

$$
\begin{aligned}
\mathbf{F} & =2 \pi a \cos (\phi)\{\overline{v h}\} \\
& \approx-2 \pi a \cos (\phi) \frac{p_{s}}{g} \mathcal{D} \partial_{y} \bar{h},
\end{aligned}
$$

with planet radius $a$, latitude $\phi$, surface pressure $p_{s}$ and gravity $g$. Here, the diffusivity $\mathcal{D}$ has units of $\mathrm{m}^{2} \mathrm{~s}^{-1}$ and can be thought of as the product of an eddy velocity $v_{e}$ 

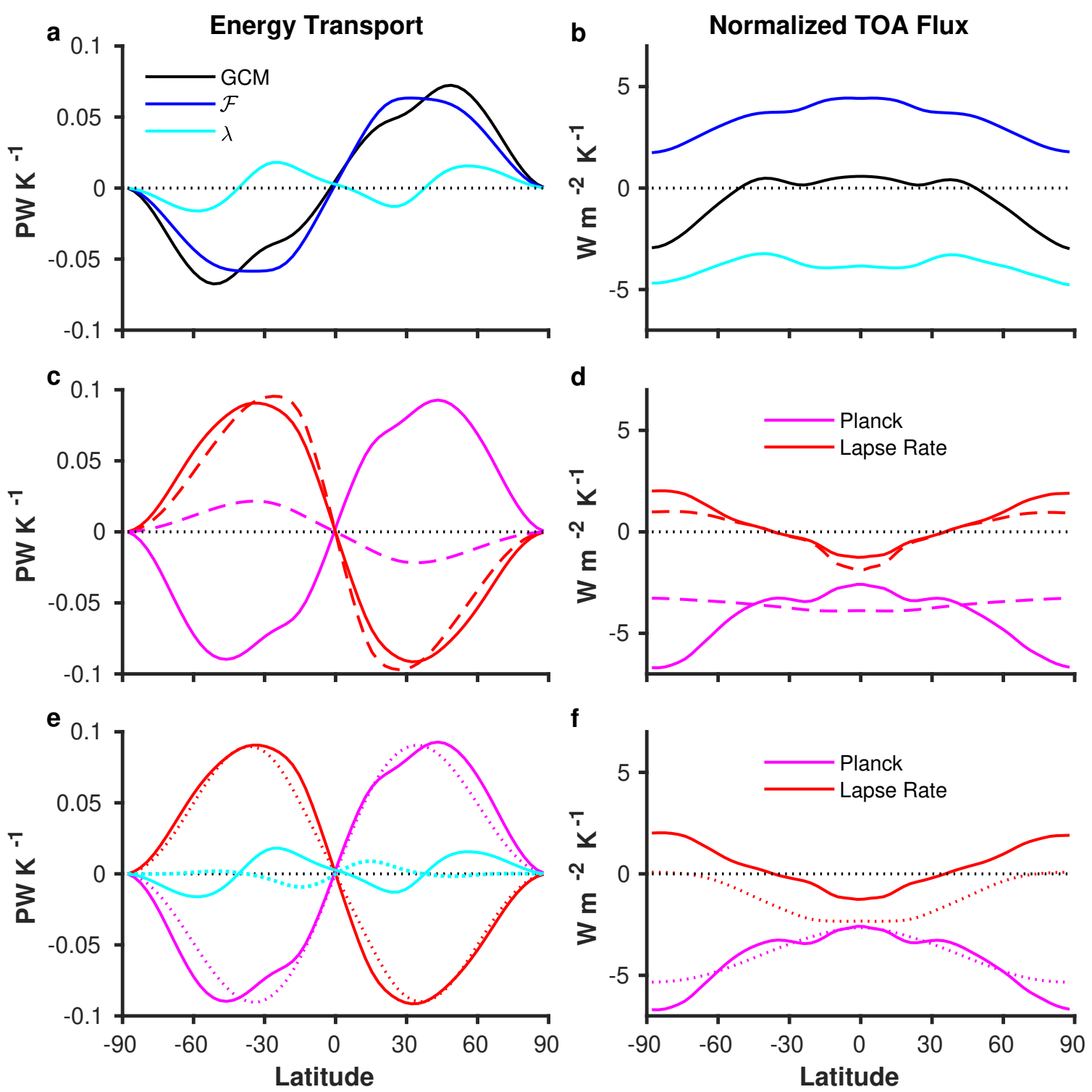

FIG. 2. (a,c,e) Change in vertically integrated northward energy transport associated with a forcing or feedback per kelvin of global warming. $(b, d, f)$ Top-of-atmosphere net radiation changes per kelvin of global warming associated with a forcing or feedback. Black lines are GCM-simulated changes, blue lines are components associated with radiative forcing, and cyan lines are components associated with the temperature feedback. Magenta and red in bottom decompose temperature feedback into Planck and lapse rate, with dashed lines corresponding to uniform warming and dotted lines corresponding to estimates derived from (9) and (10) that do not use GCM information from the warmer climate. Note that these are the normalized radiative flux changes between the Earth-like control climate and one that is $6.1 \mathrm{~K}$ warmer in the global mean; for example, the magenta line in $\mathrm{d}$ is $\left[\lambda_{P}(\phi) \times \Delta T_{S}(\phi)\right] /\left\langle\Delta T_{S}\right\rangle$.

and eddy length $\ell_{e}: \mathcal{D} \sim v_{e} \ell_{e}$. One commonly used approach is to assume the diffusivity is spatially uniform and climate invariant. That is, evaluate (5) and only allow for moist static energy (MSE) gradients $\partial_{y} \bar{h}$ to change. This is approach does not properly capture the mean-flow (e.g., Hadley circulation in the tropics) component of the energy transport, but may be adequate in the midlatitudes where the transient eddy transport (indicated by $\cdot^{\prime}$ ) dominates: $\{\overline{v h}\} \approx\left\{\overline{v^{\prime} h^{\prime}}\right\}$. Even in this simple form, there remains the challenge of characterizing changes in the mean energy gradients $\partial_{y} \bar{h}$ without knowledge of the perturbed climate. Here, we simply use diagnosed GCM fields.

Alternatively, one can account for the variation in eddy statistics that determine the diffusivity, $v_{e}$ and $\ell_{e}$, with climate. Various questions then arise:

- how to estimate the eddy statistics $v_{e}$ and $\ell_{e}$ in terms of mean fields? 
- what are the relevant averages (e.g., a vertically averaged or near-surface average of $h$ )?

- is it advantageous to use separate closures for latent energy and dry static energy transports (rather than their sum, the MSE), given the distinct behavior of condensation?

Our purpose is not to systematically address these or related questions, which are the subject of an extensive literature. Rather, we examine a closure from Bischoff and Schneider (2014), who analyzed a similar set of simulations, and a compare it to the simple, constant diffusivity one.

Bischoff and Schneider (2014) presented the following scaling:

$$
\begin{gathered}
\left\{\overline{v^{\prime} h^{\prime}}\right\} \approx \alpha v_{e} \Delta_{h} T\left(1+\beta q_{s}\right), \\
\alpha=c_{d} c_{p} p_{0} \cos \left(\phi_{s}\right) / g, \text { and } \\
\beta=\frac{c_{m}}{c_{d}} \frac{L_{v}}{c_{p}}\left(\frac{\epsilon_{r} L_{v}}{c_{p} T}-1\right) \mathcal{H} \hat{\Gamma},
\end{gathered}
$$

with dry and moist fitting coefficients $c_{d}$ and $c_{m}$, latent heat of vaporization $L_{v}$, ratio of dry air and water vapor gas constants $\epsilon_{r}$, storm track latitude $\phi_{s}$, saturation specific humidity $q_{s}$, relative humidity $\mathcal{H}$, and inverse static stability parameter $\hat{\Gamma}^{-1}=-p_{0} \partial_{p} \theta$ with $p_{0}=10^{5} \mathrm{~Pa}$, and other constants with their standard meaning. The humidities are evaluated in the subtropics (at $20^{\circ}$ latitude), as this is the equatorward side of the isentropic advection of moist air in baroclinic eddies. The inverse dry static stability parameter $\hat{\Gamma}$ appears in the scaling because the isentropic slope can change with climate, and a lower slope (higher dry stability, lower $\hat{\Gamma}$ ) implies weaker meridional moisture gradients along the isentrope, and therefore the same eddy velocities would have less poleward water vapor transport.

The eddy velocity $v_{e}$ is taken as the square root of the eddy kinetic energy and the storm track latitude $\phi_{s}$ is the latitude of maximum eddy kinetic energy. The meridional temperature contrast $\Delta_{h} T$ is a subtropics-to-subpolar (evaluated at $20^{\circ}$ and $70^{\circ}$ latitude) difference, and the static stability parameter is evaluated over the extratropics (over latitudes $30^{\circ}$ to $90^{\circ}$ ). All quantities, aside from the eddy kinetic energy, are vertically averaged over the near-surface, from $\sigma=0.75$ to $\sigma=0.9$ after spline interpolation (Bischoff and Schneider 2018).

\section{Energetic Perspective}

\section{a. GCM-simulated changes}

From the Earth-like control climate to one that is $6.1 \mathrm{~K}$ warmer, Figure $2 \mathrm{~b}$ shows the change in GCM-simulated TOA net radiation per kelvin of warming $\Delta N_{T O A} \times \Delta\langle T\rangle^{-1}$, the TOA radiative forcing per kelvin warming $\mathcal{F}(\phi) \times$ $\Delta\langle T\rangle^{-1}$, and the feedback-related change in net radiation per kelvin warming $\Delta N_{T O A, T} \times \Delta\langle T\rangle^{-1}$. The forcing (blue line in Fig. 2b) is larger in the tropics, as is the case for $\mathrm{CO}_{2}$ forcing estimates based on comprehensive climate models or reanalysis meteorological fields (Huang and Zhang 2014; Huang et al. 2016). The equator-to-pole structure of the radiative forcing results from the climatological temperature distribution, including the lapse rate (Huang et al. 2016; Jeevanjee et al. 2021). This forcing structure provokes an increase in the poleward energy transport (3) (blue line in Fig. 2a). The TOA flux change from radiative feedbacks in this idealized GCM has a more subtle spatial structure with both tropical and polar regions being more stabilizing than midlatitudes (cyan line in Fig. 2b). This mandates a decrease in poleward transport from the tropics to the midlatitudes and then an increase in transport from the midlatitudes to the poles (cyan line in Fig. 2a, eqn. 3).

The feedback-induced changes in TOA net radiation can be decomposed into Planck and lapse rate components. These feedback parameters, shown in Fig. 3a and c, have spatial structure that broadly matches that found in comprehensive GCMs (black dot-dashed vs colored lines in Fig. 3a,c). First, the Planck feedback is stabilizing everywhere (Fig. 3a) and would lead to a larger increase in the outgoing longwave radiation in low latitudes if the warming was spatially uniform. Second, the lapse rate feedback is stabilizing in low latitudes and destabilizing in high latitudes [Fig. 3c, see Henry and Merlis (2019) for a detailed discussion of this GCM's lapse rate feedback structure]. The effect of these temperature feedback components on the equilibrated change in net radiation and energy transport depends on the product of the feedback parameter and the temperature change pattern. The warming pattern is polar amplified, with about a factor of 2 more warming at the pole than in the global mean (Fig. 3e). The magnitude of the polar amplification more than offsets the Planck feedback's structure, which varies by $\approx 20 \%$. The Planck component of $\triangle N_{T O A}$ produces a larger negative TOA flux change in high latitudes than in low latitudes (magenta line in Fig. 2d). This Planck feedback change in the TOA radiation mandates an increase in the poleward energy transport (magenta line in Fig. 2c). The lapse rate feedback's change in the TOA radiation largely follows the structure of the feedback parameter: there is a relative increase in outgoing longwave in low latitudes and a relative decrease in high latitudes (red line in Fig. 2d). This energetic change mandates a decrease in the poleward energy transport (red line in Fig. 2c). The magnitudes of these individual components of the energy transport changes from the temperature feedback are comparable and offset each other to a large degree, so that the net feedback has a relatively small change compared to the radiative forcing (cyan vs. blue lines in Fig. 2a).

Figure 4a shows that the structure of the radiative forcing requires an increase in energy transport in the mid-latitudes across the range of climates (blue), while the feedback accounts for the non-monotonic behavior (cyan). The radia- 

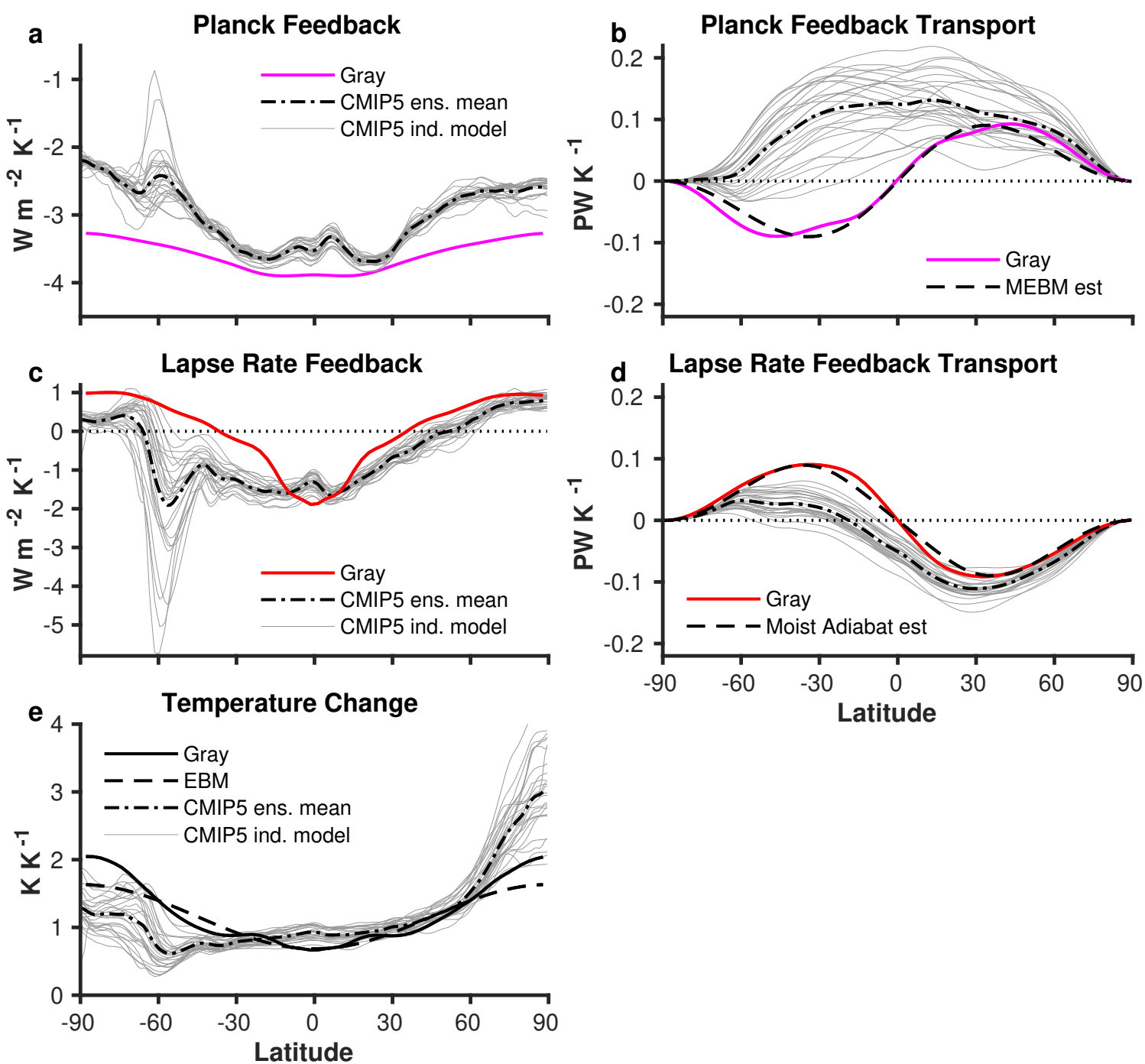

FIG. 3. Planck feedback (a) parameter and (b) the associated energy transport for the gray radiation GCM (magenta line), CMIP5 ensemble mean (black dot-dashed line), individual CMIP5 models (thin gray lines), and the moist energy balance model estimate for the transport (black dashed line). Lapse rate feedback (c) parameter and (d) the associated energy transport for the gray radiation GCM (red line), CMIP5 ensemble mean (black dot-dashed line), individual CMIP5 models (thin gray lines), and the moist adiabat estimate for the transport (black dashed line). (e) Normalized surface air temperature change $\left(\Delta T_{S}(\phi) \times\left\langle\Delta T_{S}\right\rangle^{-1}\right)$ for the gray radiation GCM (black solid), moist energy balance model theory (black dashed) CMIP5 ensemble mean (black dashed dotted), individual CMIP5 models (thin gray lines). All gray radiation GCM simulations are evaluated between the control and one that is $6.1 \mathrm{~K}$ warmer in the global mean. All CMIP5 results use the $4 \times \mathrm{CO}_{2}$ scenario.

tive forcing gives a rate of increase that is $0.06 \mathrm{PW}$ per $\mathrm{K}$ or $1.4 \%$ per $\mathrm{K}$ over the range of climates. The control simulation has a comparable rate of forcing-related change in energy transport, and there are higher rates in cold than in warm climates. To understand the temperature feedback's role in the non-monotonic behavior, it is helpful to decompose it into lapse rate and Planck components. We further investigate the influence of the warming pattern on the feedback-related transport changes by considering the following cases: the full climate-state dependent temperature change, a globally uniform temperature change that varies with climate state, and a fixed pattern of tem- perature change that scales linearly with the global-mean temperature change.

The Planck feedback is generally associated with an increase in transport with warming (magenta circles in Fig. 4b). As was the case for the smaller perturbation about the Earth-like reference, this arises from the structure of $\Delta T_{s}: \lambda_{P}(\phi) \Delta T_{s}(\phi)$. The Planck feedback $\lambda_{P}(\phi)$ with a uniform warming would decrease poleward energy transport (magenta dashed line in Fig. 4c). In addition to considering a uniform warming pattern, we can assess the energy transport changes that would be induced if the pattern of warming was unchanged (i.e., if the pattern were 

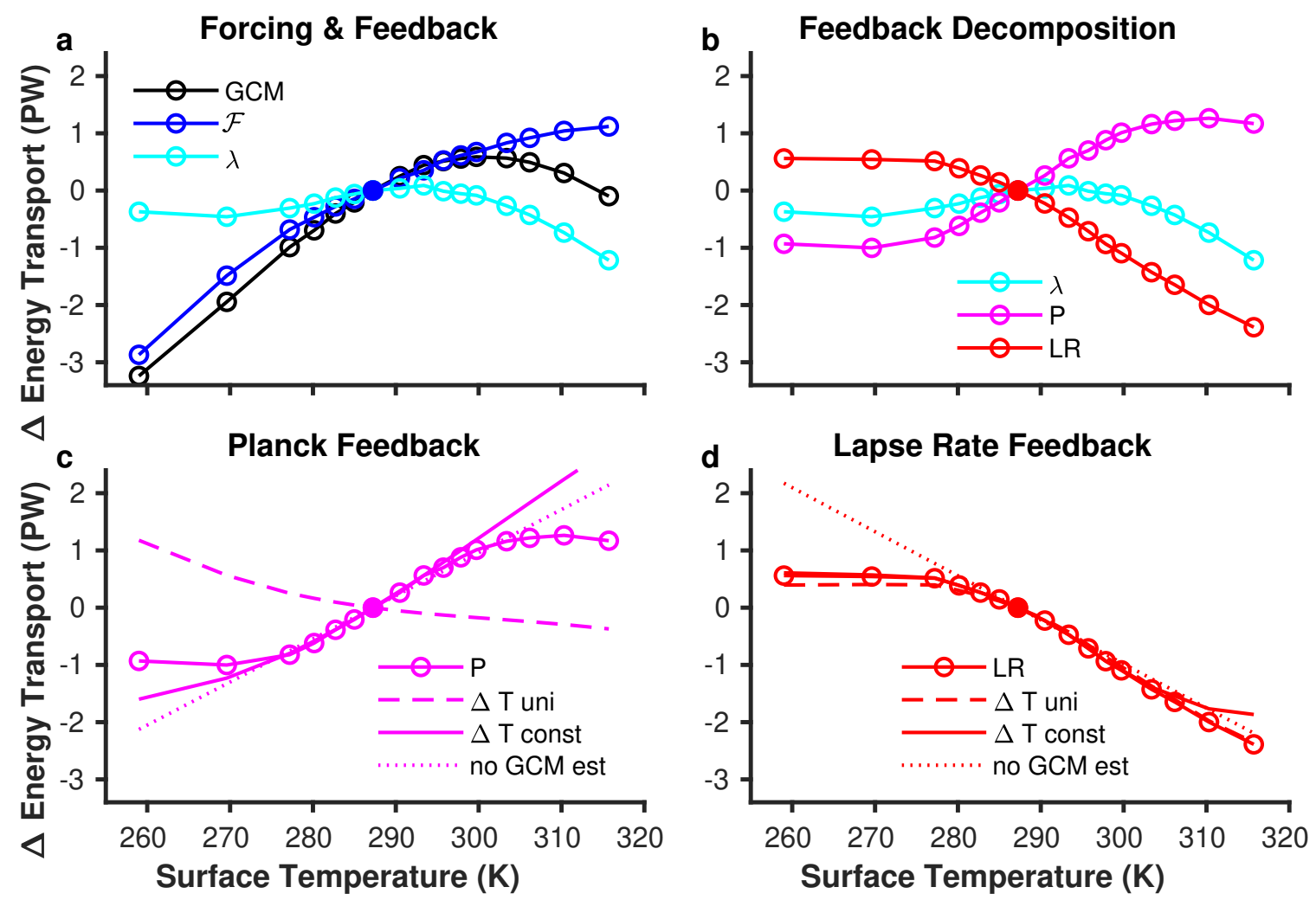

FIG. 4. (a) Change in vertically integrated northward energy transport at $50^{\circ} \mathrm{N}$ with (blue) forcing and (cyan) feedback components. (b) Decomposition of the feedback-related energy transport into (magenta) Planck and (red) lapse rate feedback components. Top-of-atmosphere energy budget changes. (c) Planck feedback computed using the climate-state dependent temperature change (magenta circles), a uniform temperature change (magenta dashed), a fixed temperature change pattern with varied amplitude (magenta solid), and a temperature change pattern from a moist energy balance model (magenta dotted). (d) Lapse rate feedback computed using the climate-state dependent temperature change (red circles), a uniform temperature change (red dashed), a fixed temperature change pattern with varied amplitude (magenta solid), and a moist adiabatic temperature change with uniform surface warming (red dotted).

always the same as that shown in Fig. 3e) over the range of climates, but scaled to match the global-mean temperature change (magenta solid line in Fig. 4c). This has a slope of $\approx 0.09 \mathrm{PW}$ per $\mathrm{K}$, with some differences in the coldest climates that result from changes in the feedback parameter. For the warmest climates, the pattern of temperature change evolves to be more spatially uniform (Fig. 5e) such that the Planck feedback-related change in energy transport is modest for climates with temperatures $\gtrsim 300 \mathrm{~K}$ (magenta circles vs. magenta solid in Fig. 4c). The temperature change pattern is also more spatially uniform in the coldest climates (dark blue line in Fig. 5e), which accounts for the modest Planck-feedback related change in energy transport.

The lapse rate feedback generally provokes decreases in poleward transport with warming across the range of climates, with weak changes in the coldest climate states (red circles in Fig. 4b). This is from the weaker meridional structure of $\lambda_{l r}$ (Fig. 5c) and would be similar with a uniform surface temperature change pattern (red solid and dashed lines nearly coincide with the red circles in Fig. 4d).

According to the energetic perspective, the radiative forcing mandates increased energy transport at midlatitudes for positive radiative forcing. The feedback-related energy transport tends to increase from cold to Earth-like climates and decrease it for sufficiently warm climates. The origin of the non-monotonicity of mid-latitude energy transport arises from: i) larger increases with warming in Planck feedback-related transport than lapse rate feedback-related decreases in cold climates, ii) near-exact cancellation from temperature feedback components in Earth-like climates, and iii) the Planck feedback-related transport asymptoting in hot climates while the lapse rate feedback-related transport consistently decreases over the whole range of climates.

\section{b. GCM-independent theories}

Having diagnosed how the energy budget changes depend on the feedback parameter and temperature change 
pattern, we explore "a priori" arguments that would constrain these quantities without knowledge of the GCMsimulated perturbed climate states.

\section{1) Moist EBM theory for Planck feedback transport}

The polar amplified warming pattern is important for the Planck-feedback related energy transport increase with warming. Here, we attempt to estimate the warming pattern using moist energy balance model (EBM) theory (Merlis and Henry 2018). The theory is an approximate solution for the temperature sensitivity of the second Legendre polynomial component $P_{2}$ (subscript indicates Legendre polynomial order) of the surface temperature. It is derived from the equilibrium of the constant diffusivity $\mathcal{D}$, constant forcing $\mathcal{F}$, constant feedback parameter $\lambda$, EBM governing equation: $0=\mathcal{F}+\lambda \Delta T-\partial_{x}\left[\mathcal{D}\left(1-x^{2}\right) \partial_{x} \Delta h\right]$, where $T$ and $h$ are surface variables and the coordinate is $x=\sin (\phi)$. The essence of the theory is to assume a uniform warming to estimate the increase in latent energy transport and solve for the weaker temperature gradient state that will balance the energy budget via both longwave radiation and a change in the transport that depends on temperature gradients.

For a one-kelvin global warming, eqn. 14 of Merlis and Henry (2018) leads to the formula for an estimate of the temperature change $\Delta T_{E B M}$ :

$$
\Delta T_{E B M}=\left[\frac{-\left.6 T_{2} \mathcal{D} L \mathcal{H} c_{p}^{-1} \partial_{T T} q^{*}\right|_{\langle T\rangle}}{6 \mathcal{D}^{\prime}-\lambda}\right] P_{2}+1,
$$

with climatological temperature $T_{2}=30 \mathrm{~K}$, relative humidity $\mathcal{H}=0.8$, diffusivity $\mathcal{D}=0.3 \mathrm{~W} \mathrm{~m}^{-2} \mathrm{~K}^{-1}$, diffusivity modified by climatological humidity $\mathcal{D}^{\prime}=0.6 \mathrm{~W} \mathrm{~m}^{-2} \mathrm{~K}^{-1}$, second temperature derivative of saturation specific humidity evaluated at the global-mean surface temperature $\left.\partial_{T T} q^{*}\right|_{\langle T\rangle}=2.3 \times 10^{-5} \mathrm{~K}^{-2}$, longwave radiative feedback parameter $\lambda=-3.7 \mathrm{~W} \mathrm{~m}^{-2} \mathrm{~K}^{-1}$, and latent heat of vaporization $L=2.5 \times 10^{6} \mathrm{~J} \mathrm{~kg}^{-1}$, and heat capacity at constant pressure $c_{p}=1004.6 \mathrm{~J} \mathrm{~K}^{-1} \mathrm{~kg}^{-1}$. These are characteristic values, rather than detailed fits to the idealized GCM, and we note that this diffusivity is related to the kinematic diffusivity (with units of $\mathrm{m}^{2} \mathrm{~s}^{-1}$ ) described in section 3 by the atmospheric mass $p_{s} / g$. The moist EBM pattern of surface temperature change is shown in Fig. 3e in the black dashed line.

Using the results of this theory to estimate the warming pattern has the virtue of requiring only control-climate information. There is, however, an extent to which the theory's success should be considered fortuitous: an analysis of this GCM's regional energy budget changes shows that the lapse rate feedback-neglected from the moist EBM theory-tends to enhance polar warming (Henry and Merlis 2019). A contravening factor, which is also neglected here, is that the spatial pattern of the radiative forcing is tropically amplified, and this tends to decrease polar amplification by decreasing the numerator of the term in square brackets in (9).

Figure 2e,f shows the implied energy transport and TOA net radiation using the GCM-independent $\triangle T_{E B M}$. There is substantial agreement. Holding this warming pattern fixed and varying the amplitude according to the magnitude of global-mean surface temperature change captures the Planck feedback-related energy transport changes at $50^{\circ}$ well over the range of climates with global-mean surface temperatures ranging from 270 to $300 \mathrm{~K}$ (Fig. 4c). As documented using the fixed pattern of warming obtained from the GCM, these energy transport changes in the coldest and hottest climates require information about the evolving pattern of warming.

The EBM theory does anticipate weaker polar amplified warming in climates with low global-mean surface temperature because there is less of a change in latent energy [as $\left.\partial_{T T} q^{*}\right|_{\langle T\rangle}$ in (9) is small for cold $\left.\langle T\rangle\right]$. It also anticipates that warming hot climates with weak temperature gradients will have less polar amplified warming [as $T_{2}$ in (9) is small for hot climates]. There are competing changes (e.g., hot climates have larger magnitude $\left.\partial_{T T} q^{*}\right|_{\langle T\rangle}$ ), so producing a closed theory for the evolution of the warming pattern through the range of climates is difficult.

Finally, we note that this moist EBM temperature change pattern is derived assuming a constant diffusivity, yet the diffusivity changes are important for energy transport changes (Fig. 1b). This is an apparent contradiction; however, the moist EBM will still produce a polar amplified temperature change pattern if the diffusivity is temperature dependent, provided the diffusivity does not decrease too rapidly with temperature. Furthermore, the assessment of the underlying diffusive closure in the GCM shown in Fig. $1 \mathrm{~b}$ is suggestive of a nominally increasing diffusivity with warming at the control climate, with that evaluation of the mean energy gradient. (This stands in contrast to CMIP5 models analyzed by Armour et al. (2019); while there is an increased MSE contrast from the subtropics to the subpolar region in the idealized GCM, the local midlatitude gradients do not behave in the same way.)

\section{2) Moist adiabat THEORY FOR LAPSE RATE FEEDbaCK TRANSPORT}

The lapse rate feedback has structure that arises from the vertically inhomogeneous distribution of temperature change and from the inhomogeneous distribution of absorbers in the atmosphere. Here, we approximate the temperature change from moist adiabats and use the radiative kernel technique (Soden et al. 2008) to quantify how this affects the temperature feedback relative to a vertically uniform temperature change.

In this idealized GCM, the structure of the atmospheric temperature and its responses to warming are shaped by a combination of large-scale flows coupled to latent heat 
release (e.g., O'Gorman 2011), radiative tendencies (e.g., Henry and Merlis 2019; Henry et al. 2021), and sub-grid scale parameterizations, particularly moist convection in the tropics (e.g., Frierson 2007; Schneider and O'Gorman 2008). A starting point for the inhomogeneous vertical structure of warming is to consider how moist adiabats lifted from the control simulation's surface temperature change in response to a uniform warming $\Delta T_{m a}$, though this neglects the important roles of the pattern of surface warming, moist eddies in the extratropics, and radiative changes in polar regions.

The change in net radiation at TOA is the product of the radiative kernel and the temperature change at each discrete level $k: \Delta N_{T O A}=\sum_{k} K_{T} \Delta T_{k}$, where $K_{T}=\partial_{T_{k}} N_{T O A}$ is the temperature kernel. We use the offline radiative transfer code implemented by Qun Liu to construct the temperature kernel for the control simulation (Liu 2020). The GCMindependent estimate of the lapse rate feedback's TOA flux is then the vertical integrated $K_{T} \times \Delta T_{m a}$ minus that of a uniform warming:

$$
\Delta N_{T O A, l r} \approx K_{T} \times \Delta T_{m a}-K_{T} \times \Delta T_{u n i} .
$$

This can be converted to an energy transport following (3).

Figure 2e,f shows the moist adiabatic estimate (10) of the lapse rate feedback. The TOA net radiation change is systematically more stabilizing (negative) than the GCMsimulated changes (red solid vs. red dotted in Fig. 2f). This is a global-mean bias that is removed when the TOA flux change is converted to an energy transport change (3). The energy transport change by this estimate of the lapse rate feedback matches the diagnosed lapse-rate feedbackrelated energy transport well (dotted vs. solid red lines in Fig. 2e). The near-exact correspondence in the extratropics results from small canceling errors: the uniform surface warming starting point for this GCM-independent estimate should lead to a smaller magnitude change (red dashed line in Fig. 2c), but this is offset by some correspondingly modest error in the spatial structure of the implied feedback parameter. Over the range of climates, this estimate, rescaled by the varying global-mean temperature changes, captures the changes at $50^{\circ}$ latitude for climates with global-mean surface temperatures ranging from 280 to $315 \mathrm{~K}$ (dotted line in Fig. 4d).

For cold climates, the feedback parameter itself is changing in an important way. Figure $5 \mathrm{c}$ shows the lapse rate feedback parameter vs. latitude for a subset of climate states. The local minimum in the deep tropics decreases with cooling and the high latitude local maxima also decreases. The less stabilizing tropical lapse rate feedback in cold climates is consistent with the cold absolute temperatures leading to less humidity to condense and warm the mid- and upper-troposphere. We have not assessed the moist adiabat argument quantitatively (i.e., with different $\Delta T_{m a}$ and kernels) in this cold regime.

\section{3) COMBined TEMPERATURE FEEDBACK TRANSPORT}

Taking the two components of the temperature feedback together, there is a large degree of cancellation near the control climate. Do these two GCM-independent estimates have similar behavior? Their sum is substantially less than the individual changes (dotted cyan line in Fig. 2e). The estimate has the opposite sign in both the low latitudes (increase rather than decrease in poleward transport) and the extratropics (weak decrease rather than increase in poleward transport). In summary, these GCM-independent estimates are successful in leading to the expectation that the forcing-related energy transport change dominates the feedback-related changes.

\section{c. Comparison to comprehensive GCMs}

\section{1) CMIP5}

Figure 3 shows the Planck and lapse rate feedbacks and the associated energy transports with the temperature change pattern for CMIP5 GCMs, as well as the idealized GCM simulations previously described. There is regional spread in local feedbacks and the pattern of warming between CMIP5 GCMs (gray lines in Fig. 3a,c and e) that gives rise to spread in the associated energy transports (gray lines in Fig. 3b,d), with individual models varying by a factor of $\sim 3$ in the Planck feedback-related energy transport and by of $\sim 2$ in the lapse rate feedback-related energy transport. The temperature change pattern in CMIP5 features the well known Arctic amplification (about a factor of 3 more warming than the global mean) and a Southern Ocean region of muted temperature change (the temperature change in southern hemisphere high latitudes is comparable to the global mean), with substantial inter-model spread in Arctic amplification. The hemispheric symmetry of the idealized GCM's statistical steady state is polar amplified with about a factor of 2 more warming near the pole. As we proceed, we focus on the comparison between the ensemble mean CMIP5 results and the idealized GCM.

The CMIP5 and idealized GCM Planck feedback latitudinal structure is broadly similar: there is a local minimum in the tropics that is about $1 \mathrm{~W} \mathrm{~m}^{-2} \mathrm{~K}^{-1}$ more negative than in the polar regions (Fig. 3a). The CMIP5 Planck feedback is a less stabilizing global-mean feedback owing to the climatological clouds that lower the emission temperature and there is interhemipheric asymmetry, with the southern hemisphere's colder high latitudes giving rise to a larger equator-to-pole contrast, that is absent in the idealized GCM. The CMIP5 Planck feedback-related energy transport is northward at all latitudes (Fig. 3b). That is, it is increasing the poleward transport in the northern hemisphere and decreasing it in in the southern hemisphere. The northern hemisphere extratropics are similar to both the idealized GCM and the moist EBM estimate. The northward change in transport in the southern hemisphere 
is consistent with a nearly flat warming pattern and the feedback parameter that is less negative in high latitudes (akin to the previous discussion of what the idealized GCM's Planck feedback-related transport would be if there was a uniform temperature change).

The CMIP5 lapse rate feedback parameter has a spatial structure that increases from a stabilizing value of $\approx-1.7 \mathrm{~W} \mathrm{~m}^{-2} \mathrm{~K}^{-1}$ to destabilizing values of near $\approx 1.0 \mathrm{~W} \mathrm{~m}^{-2} \mathrm{~K}^{-1}$ in the northern hemisphere and $\approx$ $0.4 \mathrm{~W} \mathrm{~m}^{-2} \mathrm{~K}^{-1}$ in the southern hemisphere polar regions, poleward of a local minimum over the Southern Ocean (Fig. 3b) where large-scale ocean upwelling reduces surface warming and decouples the surface from the tropospheric response (Po-Chedley et al. 2018). The idealized GCM has equator-to-pole contrasts comparable to the CMIP5 ensemble mean's northern hemisphere, but the zero crossing from stabilizing to destabilizing occurs about $10^{\circ}$ in latitude equatorward. The CMIP5 lapse rate feedbackrelated energy transport is a decrease in poleward transport in both hemispheres (Fig. 3d): in the southern hemisphere midlatitudes, the decrease is about a factor of 3 smaller than than that of the northern hemisphere. Given that the lapse rate feedback-related transport is less sensitive to the warming pattern because the feedback parameter changes sign, this suggests that the feedback parameter's Southern Ocean local minima-flattening the overall hemisphere's feedback - is playing an important role in moderating that hemisphere's decrease in transport. As in the case of the Planck feedback-related transport change, the CMIP5 lapse rate feedback energy transport change and idealized GCM estimate are similar in the northern hemisphere extratropics.

We also show the GCM-independent estimates for the energy transport from the Planck and lapse rate feedbacks (black dashed lines in Fig. 3b,d). These are comparable to the ensemble-mean CMIP5 changes in the northern hemisphere. This is encouraging in that the GCM-independent estimates rely on basic aspects of the climatology that are similar between the idealized GCM and CMIP5 models. For example, the moist EBM theory depends on the global-mean temperature and equator-to-pole temperature contrast and the moist adiabatic estimate depends on the surface temperature, which are similar between comprehensive and idealized GCMs. We also expect that the form of the moist EBM estimate that we present does not account for the muted southern hemisphere temperature change given that we did not prescribe the ocean's surface flux. Finally, accounting for the relevant structure of the southern hemisphere lapse rate feedback is beyond the scope of the simple moist adiabatic estimate. In summary, there are meaningful discrepancies between the GCM-independent theories and CMIP5 simulations in the southern hemisphere and a large degree of agreement in the northern hemisphere.

\section{2) $\mathrm{CCSM} 3$}

Figure 5 shows the total feedback parameter, lapse rate feedback parameter, and temperature change pattern for a subset of different climate states in the idealized GCM (left) and for the six $\mathrm{CO}_{2}$ doublings of the CCSM3 simulations (right).

The total feedback parameter in the idealized GCM has more spatial structure with warming, provoking an increased energy transport, until the warmest climates (Fig. 5a). In contrast, the total feedback in CCSM3 has more modest and less systematic local changes, becoming somewhat more spatially uniform with warming (Fig. 5b). These differences arise from additional feedbacks, particularly shortwave feedbacks that can offset the temperature feedbacks, and these also play a role in the higher-order meridional structure of the total feedback parameter in CCSM3.

The idealized GCM has a lapse rate feedback parameter with spatial structure that is enhanced and an overall stabilizing influence that increases with warming (Fig. 5c). In the coldest climates, the lapse rate feedback parameter is nearly spatially uniform and it becomes more spatially uniform in the hottest climate shown. CCSM3 has an equatorial minimum (stabilizing) in the feedback parameter and the amplitude increases with warming until the two hottest climates (Fig. 5d). The range of tropical lapse rate feedback is smaller in CCSM3, both because the range of climates excludes ones colder than the reference and because of the behavior of the warmest simulations. There, Caballero and Huber (2013) describe substantial tropical cloud radiative feedbacks. These can interact with the lapse rate feedback via radiative influences on the tropical temperature change and through changes in cloud masking (the extent to which tropospheric temperature change affect the TOA flux depends on the cloud distribution, as comparisons of clear-sky and all-sky radiative kernels show), neither of which are present in the idealized GCM. Another interesting regional comparison is in high latitudes. The idealized GCM has a destabilizing lapse rate feedback over much of the range of climates, despite the absence of ice and ice albedo processes. [These are included in the model hierarchy described in Feldl and Merlis (2021) but only modestly affect this GCM's high latitude lapse rate feedback for a moderate magnitude of climate change, their Fig. S2.] CCSM3 has less destabilizing high-latitude lapse rate feedback as the climates progress toward warm climate states, suggestive of a decreasing role for the near-surface enhanced warming that results from sea ice or surface flux changes, which makes the atmospheric lapse rate feedback more destabilizing (e.g., Graversen et al. 2014; Feldl et al. 2020; Henry et al. 2021).

The temperature change pattern of the idealized GCM shows relatively less polar amplification in the coldest climates (about $1.5 \times$ as much warming at the pole as in the 

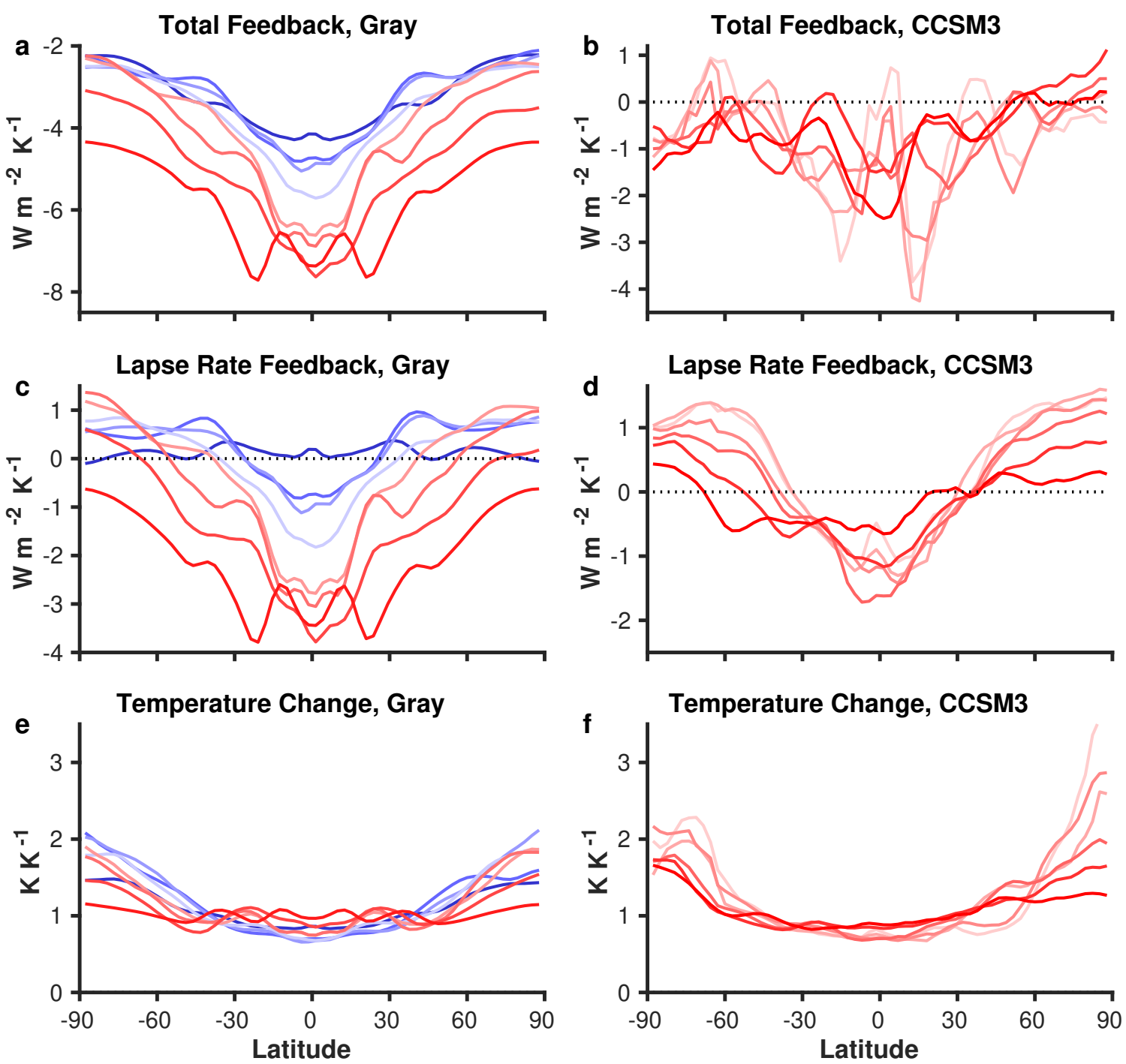

FIG. 5. Total feedback parameter for the (a) gray radiation GCM and (b) CCSM3. Lapse rate feedback parameter for the (c) gray radiation GCM and (d) CCSM3. Normalized surface air temperature change $\left(\Delta T_{S}(\phi) \times\left\langle\Delta T_{S}\right\rangle^{-1}\right)$ for the (e) gray radiation GCM and (f) CCSM3. Colors are proportional to the global-mean temperature, with darker blues coldest and darker reds warmest, and similar colors indicate similar global-mean surface temperature across the two GCMs. Note that the vertical axes change fro left to right and the idealized GCM panels show eight of the sixteen simulations for clarity.

global mean) and in the warmest climates, where the temperature change is nearly uniform in latitude. In CCSM3, there is polar amplification in all the climate states, although the Arctic amplification weakens from a factor of 2.5-3 for $2 \times$ and $4 \times$ pre-Industrial $\mathrm{CO}_{2}$ concentrations to a factor of about 1.2 in the warmest climates.

Though the presence of additional feedbacks-in particular those associated with clouds and water vapor-makes the total feedback in CCSM3 flatter than in the idealized GCM, it still has a qualitatively similar structure, with more negative values in the tropics and less negative or even positive values in the high latitudes. As a result, the total feedback together with the polar-amplified tempera- ture change implies decreasing poleward energy transport as the climate warms in CCSM3, as in the idealized GCM. Both GCMs have less polar amplification in the warmest climates than near the control. The CCSM3 simulations do not include cold climates, and it would be interesting to examine how polar amplification evolves in comprehensive simulations of cold climates.

\section{Diffusive Perspective \\ a. GCM-simulated changes}

First, we examine the simple spatially-constant-andclimate-invariant diffusivity closure (5). Figure $1 \mathrm{~b}$ shows 

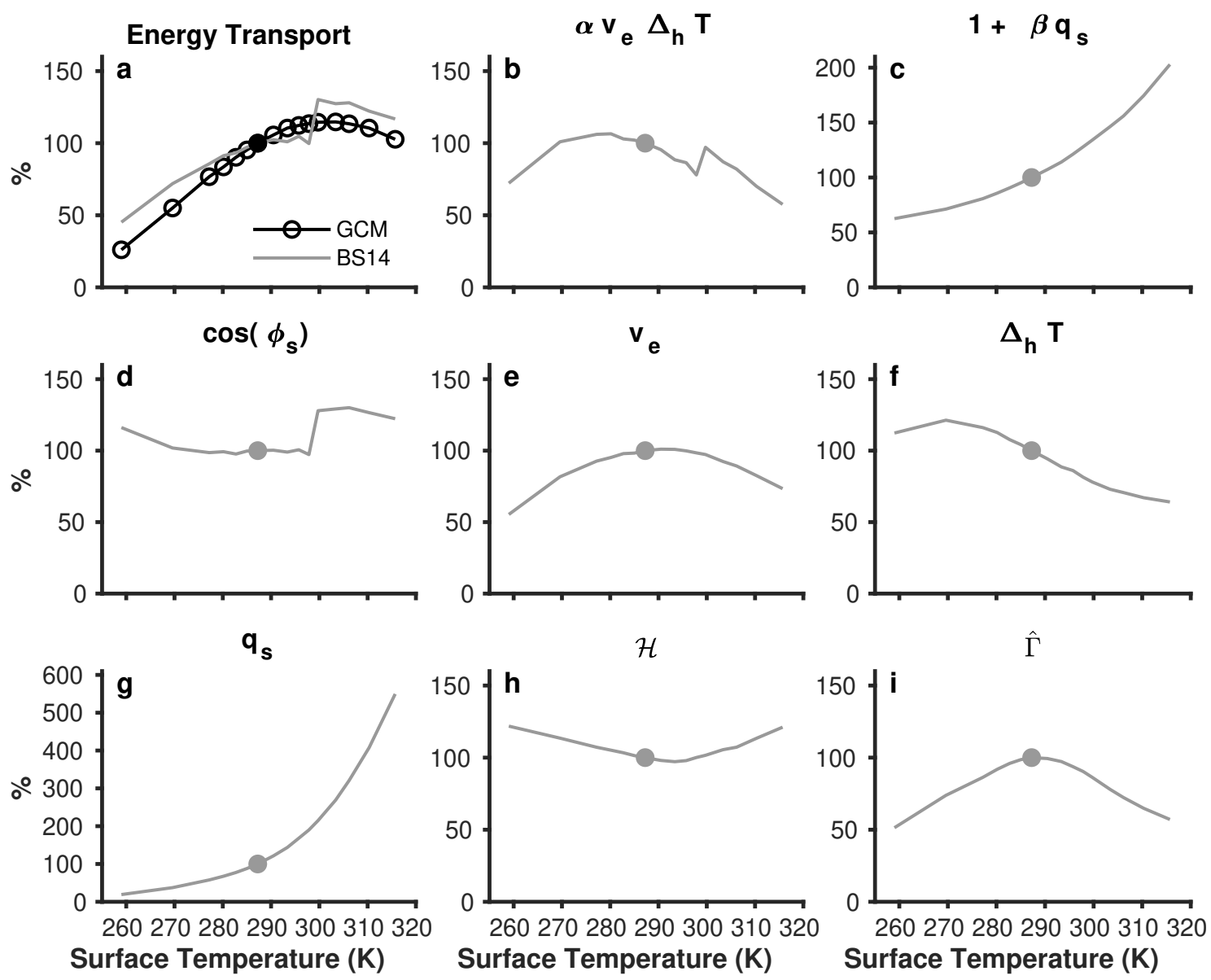

FIG. 6. (a) Percentage change in vertically integrated northward energy transport at $50^{\circ} \mathrm{N}$ for GCM simulations (black circles) and Bischoff and Schneider (2014) diffusive scaling (6) (gray) relative to the control simulation. (b) Dry component $\alpha v_{e d d y} \Delta_{h} T$ and (c) latent energy modification $1+\beta q_{s}$ of the diffusive closure. (d,e,f,g,h,i) Cosine of storm track latitude $\cos \left(\phi_{s}\right)$, eddy velocity $v_{e d d y}$, horizontal temperature contrast $\Delta_{h} T$, saturation specific humidity $q_{s}$, relative humidity $\mathcal{H}$, and inverse dry static stability parameter $\hat{\Gamma}$. The control simulation is indicated by the filled symbols, and the GCM averaging conventions are described in section 3. Note that the vertical scales differ in panels $\mathrm{c}$ and $\mathrm{g}$.

that this estimate of the mid-latitude energy transport's changes with climate does not capture the variation of the GCM. The scaling remains near the control simulation's value of roughly $4 \mathrm{PW}$ over the range of climates. Clearly, it is quantitatively important to account for climate-state dependent variations in the diffusivity, as the MSE gradients in the midlatitudes do not vary in the way the energy transport does. (An alternative simple estimate that uses the difference between the subtropical near-surface MSE and subpolar MSE has a nearly monotonic increase with temperature and implies a factor of $\approx 2$ increase in the estimated mid-latitude energy transport.)

Figure 6a shows the diffusive closure of Bischoff and Schneider (2014) (6) as a percentage relative to the control climate. This closure broadly captures aspects of the shape of the mid-latitude energy transport changes. It increases from cold climates, reaches a local maximum for climates with global-mean surface temperature near $300 \mathrm{~K}$ and decreases for warmer climates. Unlike the energetic decomposition which uses the GCM-simulated net radiation, there is no constraint that the diffusive closure match the total energy transport, and there are $\approx 25 \%$ discrepancies between the scaling and the GCM-simulated transport.

The scaling can be broken down into components associated with the various fields that enter it. First, we consider percentage changes in the term associated with the dry transport $\alpha v_{e} \Delta_{h} T$ (Fig. 6b) and the modification to that by the latent energy $1+\beta q_{s}$ (Fig. 6c):

$$
\frac{\delta\left[\alpha v_{e} \Delta T\left(1+\beta q_{s}\right)\right]}{\alpha v_{e} \Delta_{h} T\left(1+\beta q_{s}\right)} \approx \frac{\delta\left(\alpha v_{e} \Delta_{h} T\right)}{\alpha v_{e} \Delta_{h} T}+\frac{\delta\left(1+\beta q_{s}\right)}{1+\beta q_{s}},
$$

with the denominator evaluated at the control climate. The dry part of the scaling increases from the coldest climates to one somewhat colder than the reference before decreasing with warming. The modification to the scaling from latent energy monotonically increases with warming by a factor 
of $\approx 3$ over the range of climates. Each of these two components of the scaling, in turn, depend on GCM-simulated fields.

There is "dynamic" information entering the scaling via the storm track latitude $\phi_{s}$ and the eddy velocity $v_{e}$. The storm track latitude is sensitive to the averaging convention. Figure $6 \mathrm{~d}$ shows the latitude of the maximum total eddy kinetic energy gradually moves poleward with warming, slightly reducing the $\cos \left(\phi_{S}\right)$ that appears in the scaling, until an abrupt equatorward shift occurs, increasing $\cos \left(\phi_{s}\right)$, near global-mean surface temperature $300 \mathrm{~K}$. [Dwyer and O'Gorman (2017) connect an equatorward shift in the surface westerlies in these hot climates to changes in moist Eliassen-Palm fluxes.] However, if we instead use a near-surface measure of eddy kinetic energy, which is a physically plausible choice given the vertical structure of atmospheric water vapor, the latitude of the maximum nearly monotonically increases with warming (not shown). This has the effect of placing the scaling's maximum energy transport at the control climate, rather than near the climate with global-mean surface temperature $300 \mathrm{~K}$. The eddy velocity $v_{e}$ increases from the coldest climates to the Earth-like control before decreasing in warmer climates (Fig. 6e).

The meridional temperature contrast $\Delta_{h} T$ decreases with warming, aside from the coldest two climates (Fig. 6f). These factor of $\approx 2$ variations contribute to the scaling's decrease in energy transport in the warm climates and must be offset by other changes in the cold climates.

The scaling components related to the latent energy depend on the saturation specific humidity $q_{s}$, relative humidity $\mathcal{H}$, and inverse static stability parameter $\hat{\Gamma}$. The subtropical saturation specific humidity that enters the scaling increases with warming over the range of climates and has the largest range of any scaling component (Fig. $6 \mathrm{~g}$, note different vertical scale). The roughly $30 \times$ variation from coldest to warmest is a consequence of the temperature dependence of the Clausius-Clapeyron relation. The relative humidity decreases to a local minimum at a climate somewhat warmer than the control before increasing with warming (Fig. 6h). For cold climates, this is opposing the increase in $q_{s}$, muting the change. For warm climates, this tends to augment the increase in latent energy with warming compared to constant relative humidity. The inverse static stability parameter $\hat{\Gamma}$ varies non-monotonically. The dry static stability $\partial_{p} \theta$ decreases from the cold to control climate and increases from the control to warmest climates. This means that $\hat{\Gamma}$ reaches a local maximum near the control climate and its decrease with warming partly offsets the saturation specific humidity increase. Finally, there is an additional subtropical $T^{-1}$ dependence in $\beta(8)$, and its $20 \%$ decrease over the range of climates is comparable to that of the global-mean $\langle T\rangle^{-1}$. Overall, the latent energy aspect of the scaling $1+\beta q_{s}$ (Fig. 6c) has much less of an increase with warming than the saturation specific humidity (Fig. 6g).

The scaling's rate of change at the control climate is near zero, while the GCM-simulated rate of increase is $1.7 \% \mathrm{~K}$. Over the range of climates, the eddy velocity and dry static stability are non-monotonic in a way that helps account for the simulated energy transport, while the relative humidity's non-monotonic variations tend to oppose these factors. The meridional temperature contrast decreases monotonically at a less rapid rate than the Clausius-Clapeyron increase in saturate specific humidity. [Qualitatively similar results are found in comprehensive GCMs (Held and Soden 2006) and moist diffusive EBMs (Merlis and Henry 2018).] The storm track latitude's changes depend on what measure of eddy kinetic energy (total or near-surface) is used to determine the latitude where it is maximum.

\section{b. GCM-independent theories}

It is challenging to formulate the required fields for diffusive closures without GCM simulations. In the case of the constant diffusivity closure, the moist static energy distribution is needed. This is affected by the energy transport and radiative processes. We note that typical applications of moist EBMs to comprehensive simulations of climate change use detailed results from control and perturbed GCM simulations to derive spatially varying feedback parameters as input to the moist EBM. In the case of the more thorough diffusive scaling (6), there are several required fields. We briefly discuss each here.

- The storm track latitude $\phi_{s}$ is a challenge, as it depends on the spatial structure of the atmospheric macroturbulence. However, there have been some recent efforts to use diffusive EBMs to assess storm track changes (Shaw and Voigt 2016; Mbengue and Schneider 2018). We do not pursue this here because the averaging-convention sensitivity of the GCM results is so large.

- The eddy velocity in these GCM simulations has been shown to scale with mean available potential energy (MAPE): $v_{e} \sim \sqrt{M A P E}$ by O'Gorman and Schneider (2008a). They found opposing changes from weakening meridional temperature gradients and increased tropospheric depth with warming. The extratropical static stability undergoes non-monotonic changes and the control climate is near a minimum in static stability, which helps give rise to the local maximum in $v_{e}$ as lower dry stability increases MAPE. The MAPE calculation requires GCM-simulated mean fields, some of which $\left(\Delta_{h} T\right.$ and $\left.\hat{\Gamma}\right)$, also enter the energy transport scaling (6).

- The meridional temperature contrast $\Delta_{h} T$ could be estimated using a moist EBM. We pursued this in 
section 4 . There, we noted that there are competing sensitivities in the EBM theory that currently foreclose a GCM-independent prediction of the weakening of the polar amplified temperature change pattern in the extreme (hot or cold) climates.

- An attempt to estimate the inverse dry static stability parameter $\hat{\Gamma}$ from moist adiabats, analogous to section 4 , would have increases in dry stability over the entire range of climates, which cannot account for cold climates. O'Gorman (2011) derived an "effective" stability that captures the eddy component of latent heat release on the extratropical stratification. It successfully accounts for the non-monotonic changes over range of climates, and it requires knowledge of mean temperature fields and a scaling coefficient related to the vertical velocity distribution. This scaling coefficient's changes are not captured by the moist baroclinic instability of the mean fields, suggesting non-linear dynamics are important in determining it (O'Gorman et al. 2018).

- The saturation specific humidity $q_{s}$ has a straightforward Clausius-Clapeyron temperature dependence, and approximating the local subtropical temperature with a global mean temperature with a constant offset would be adequate.

- It is difficult to formulate a GCM-independent theory for the subtropical near-surface relative humidity $\mathcal{H}$. It depends on large-scale advection (Emanuel and Pierrehumbert 1995; Galewsky et al. 2005; Hurley and Galewsky 2010) and convective tendencies (Emanuel and Pierrehumbert 1995; Schneider et al. 2006).

\section{Conclusions}

The atmospheric energy transport response to climate change is influenced by competing factors, and quantitative assessments of these factors have utilized different perspectives. Here, we assessed the energetic (or radiative) perspective and the diffusive perspective to shed light on the midlatitude energy transports over a wide range of climates in an idealized GCM.

First, the energetic perspective offers a straight-forward interpretation of why increases in optical depth (our proxy for $\mathrm{CO}_{2}$ changes) provoke increased transport in an idealized gray radiation. The forcing is larger at low latitudes than high latitudes over the range of climates. This expands the finding of Huang and Zhang (2014) to more extreme climates. The feedback component of the energetic perspective can augment or offset the forcing-related changes, including certain parts of parameter space-hot climates-where they have magnitude sufficient to overcome the forcing-related increase and produce a net reduction in the mid-latitude transport. The temperature feedback itself has Planck and lapse rate components that tend in opposite directions, with the Planck feedback-related changes increasing the midlatitude transport and the lapse rate feedback-related changes decreasing the midlatitude transport with warming. We find that both the pattern of temperature changes, particularly for the Planck feedback, and the structure of the feedback parameter matter quantitatively. Over a range of Earth-like to hot climates, we find important differences between the idealized GCM and comprehensive CCSM3 simulations. The meridional structure of CCSM3's feedback parameter is flatter, as a result of cancellation between the temperature feedbacks and additional radiative feedbacks that the idealized GCM neglects. Nevertheless, at least in warm climates, the net effect of the all feedbacks in CCSM3 is to reduce poleward energy transport, in agreement with the idealized GCM.

The diffusive perspective can also account for the GCMsimulated midlatitude energy transport changes, provided the diffusivity varies with climate. Non-monotonic changes in the eddy velocity and dry static stability are important to the non-monotonic changes in the energy transport, as the net effect of meridional temperature gradient and latent energy changes tends to monotonically increase the transport with warming.

For both diffusive and energetic perspectives on the energy transport, mean fields are required to calculate the change in transport. For the diffusive perspective, we discussed a number of outstanding challenges to go beyond using GCM-simulated mean fields in the scaling. For the energetic perspective, we made progress by examining two a priori "GCM-independent" estimates for the meridional temperature gradient and vertically varying temperature change that capture the structure of the TOA net radiation changes, which then determine the transport associated with the Planck and lapse rate feedbacks. Importantly, these GCM-independent estimates also capture the feedback-related changes in northern hemisphere extratropical energy transport in the CMIP5 multi-model mean. Additional GCM-independent arguments for the energy transport related to other radiative feedbacks (water vapor, surface albedo, and cloud) is an important future challenge.

Data availability statement. The source code for the idealized GCM and to reproduce the figures is available at https://web.meteo.mcgill.ca/ tmerlis/code/ fms_gray_forcing_merlis_etal_rad_diff.tgz.

Acknowledgments. The authors thank Paul O'Gorman, Matthew Henry, and Tobias Bischoff for helpful discussions. TMM was supported by a NSERC Discovery 
grant and Canada Research Chair (Tier 2) and a Compute Canada/Canada Foundation for Innovation computing allocation. NF was supported by NSF award AGS1753034. RC was supported by the EU Horizon 2020 Research and Innovation Programme under grant agreement 820829 (CONSTRAIN).

\section{References}

Armour, K. C., N. Siler, A. Donohoe, and G. H. Roe, 2019: Meridional atmospheric heat transport constrained by energetics and mediated by large-scale diffusion. J. Climate, 32, 3655-3680, doi:10.1175/ JCLI-D-18-0563.1.

Bischoff, T., and T. Schneider, 2014: Energetic constraints on the position of the Intertropical Convergence Zone. J. Climate, 27, 49374951.

Bischoff, T., and T. Schneider, 2018: Corrigendum: Energetic constraints on the position of the Intertropical Convergence Zone. J. Climate, 31, 927.

Caballero, R., and J. Hanley, 2012: Midlatitude eddies, storm-track diffusivity, and poleward moisture transport in warm climates. $J$. Atmos. Sci., 69, 3237-3250.

Caballero, R., and M. Huber, 2013: State-dependent climate sensitivity in past warm climates and its implications for future climate projections. Proc. Nat. Acad. Sci., 110, 14 162-14 167.

Caballero, R., and P. L. Langen, 2005: The dynamic range of poleward energy transport in an atmospheric general circulation model. Geophys. Res. Lett., 32, L02 705, doi:10.1029/2004GL021581.

Dwyer, J. G., and P. A. O'Gorman, 2017: Moist formulations of the Eliassen-Palm flux and their connection to the surface westerlies. $J$. Atmos. Sci., 74 (2), 513-530.

Emanuel, K. A., and R. T. Pierrehumbert, 1995: Microphysical and dynamical control of tropospheric water vapor. Clouds, Chemistry, and Climate, P. J. Crutzen, and V. Ramanathan, Eds., Springer, NATO ASI Series I, Vol. 35, 17-28.

Feldl, N., S. Bordoni, and T. M. Merlis, 2017: Coupled high-latitude climate feedbacks and their impact on atmospheric heat transport. $J$. Climate, 30, 189-201.

Feldl, N., and T. M. Merlis, 2021: Polar amplification in idealized climates: the role of ice, moisture, and seasons. Geophys. Res. Lett., e2021GL094130.

Feldl, N., S. Po-Chedley, H. K. A. Singh, S. Hay, and P. J. Kushner, 2020: Sea ice and atmospheric circulation shape the high-latitude lapse rate feedback. npj Clim. Atmos. Sci., 3, 1-9.

Feldl, N., and G. H. Roe, 2013: Four perspectives on climate feedbacks. Geophys. Res. Lett., 40, 4007-4011.

Flannery, B. P., 1983: Energy balance models incorporating transport of thermal and latent energy. J. Atmos. Sci., 41, 414-421.

Frierson, D. M. W., 2007: The dynamics of idealized convection schemes and their effect on the zonally averaged tropical circulation. J. Atmos. Sci., 64, 1959-1976.

Frierson, D. M. W., I. M. Held, and P. Zurita-Gotor, 2006: A grayradiation aquaplanet moist GCM. Part I: Static stability and eddy scale. J. Atmos. Sci., 63, 2548-2566.
Galewsky, J., A. Sobel, and I. Held, 2005: Diagnosis of subtropical humidity dynamics using tracers of last saturation. J. Atmos. Sci., 62 , 3353-3367.

Graversen, R. G., P. L. Langen, and T. Mauritsen, 2014: Polar amplification in CCSM4: Contributions from the lapse rate and the surface albedo feedbacks. J. Climate, 27, 4433-4450.

Hartmann, D., 1994: Global Physical Climatology. Academic Press, $411 \mathrm{pp}$.

Held, I. M., and B. J. Soden, 2006: Robust responses of the hydrological cycle to global warming. J. Climate, 19, 5686-5699.

Henry, M., and T. M. Merlis, 2019: The role of the nonlinearity of the Stefan-Boltzmann law on the structure of radiatively forced temperature change. J. Climate, 32, 335-348.

Henry, M., T. M. Merlis, N. J. Lutsko, and B. E. J. Rose, 2021: Decomposing the drivers of polar amplification with a single column model. J. Climate, 34, 2355-2365.

Huang, Y., X. Tan, and Y. Xia, 2016: Inhomogeneous radiative forcing of homogeneous greenhouse gases. J. Geophys. Res., 121, 2780-2789.

Huang, Y., and M. Zhang, 2014: The implication of radiative forcing for poleward energy transport. Geophys. Res. Lett., 41, 1665-1672.

Hurley, J. V., and J. Galewsky, 2010: A last-saturation diagnosis of subtropical water vapor response to global warming. Geophys. Res. Lett., 37 (6).

Hwang, Y.-T., D. M. W. Frierson, and J. E. Kay, 2011a: Coupling between Arctic feedbacks and changes in poleward energy transport. Geophys. Res. Lett., 38, L17 704.

Hwang, Y.-T., D. M. W. Frierson, B. J. Soden, and I. M. Held, 2011b: CORRIGENDUM: Corrigendum for Held and Soden (2006). J. Climate, 24, 1559-1560.

Jeevanjee, N., P. Hassanzadeh, S. Hill, and A. Sheshadri, 2017: A perspective on climate model hierarchies. J. Adv. Model. Earth Syst., 1760-1771.

Jeevanjee, N., J. Seeley, D. Paynter, and S. Fueglistaler, 2021: An analytical model for spatially varying clear-sky $\mathrm{CO}_{2}$ forcing. Earth and Space Science Open Archive ESSOAr.

Liu, Q., 2020: Input data for Isca radiative kernel offline calculation. Zenodo, doi:10.5281/zenodo.4071837.

Maher, P., and Coauthors, 2019: Model hierarchies for understanding atmospheric circulation. Rev. Geophys., 57, 250-280.

Mbengue, C., and T. Schneider, 2018: Linking Hadley circulation and storm tracks in a conceptual model of the atmospheric energy balance. J. Atmos. Sci., 75 (3), 841-856.

Merlis, T. M., 2014: Interacting components of the top-of-atmosphere energy balance affect changes in regional surface temperature. Geophys. Res. Lett., 41, 7291-7297.

Merlis, T. M., and M. Henry, 2018: Simple estimates of polar amplification in moist diffusive energy balance models. J. Climate, 31, $5811-5824$.

Merlis, T. M., and T. Schneider, 2011: Changes in zonal surface temperature gradients and Walker circulations in a wide range of climates. J. Climate, 24, 4757-4768. 
North, G. R., 1975: Theory of energy-balance climate models. J. Atmos. Sci., 32, 2033-2043.

O'Gorman, P. A., 2011: The effective static stability experienced by eddies in a moist atmosphere. J. Atmos. Sci., 68, 75-90.

O'Gorman, P. A., T. M. Merlis, and M. S. Singh, 2018: Increase in the skewness of extratropical vertical velocities with climate warming: fully nonlinear simulations versus moist baroclinic instability. Quart. J. Roy. Meteor. Soc., 144 (710), 208-217.

O'Gorman, P. A., and T. Schneider, 2008a: Energy of midlatitude transient eddies in idealized simulations of changed climates. J. Climate, 21, 5797-5806.

O'Gorman, P. A., and T. Schneider, 2008b: The hydrological cycle over a wide range of climates simulated with an idealized GCM. $J$. Climate, 21, 3815-3832.

Pendergrass, A. G., A. Conley, and F. M. Vitt, 2018: Surface and topof-atmosphere radiative feedback kernels for CESM-CAM5. Earth Syst. Sci. Data, 10, 317-324.

Po-Chedley, S., K. C. Armour, C. M. Bitz, M. D. Zelinka, B. D. Santer, and Q. Fu, 2018: Sources of intermodel spread in the lapse rate and water vapor feedbacks. J. Climate, 31, 3187-3206.

Roe, G., 2009: Feedbacks, timescales, and seeing red. Ann. Rev. Earth Planet. Sci., 37, 93-115.

Rose, B. E. J., K. C. Armour, D. S. Battisti, N. Feldl, and D. D. B. Koll, 2014: The dependence of transient climate sensitivity and radiative feedbacks on the spatial pattern of ocean heat uptake. Geophys. Res. Lett., 41, 1071-1078.

Schneider, T., and P. A. O'Gorman, 2008: Moist convection and the thermal stratification of the extratropical troposphere. J. Atmos. Sci., 65, 3571-3583.

Schneider, T., K. L. Smith, P. A. O'Gorman, and C. C. Walker, 2006: A climatology of tropospheric zonal-mean water vapor fields and fluxes in isentropic coordinates. J. Climate, 19, 5918-5933.

Schneider, T., and C. C. Walker, 2008: Scaling laws and regime transitions of macroturbulence in dry atmospheres. J. Atmos. Sci., 65, 2153-2173.

Sellers, W. D., 1969: A global climatic model based on the energy balance of the Earth-atmosphere system. J. Appl. Meteor., 8, 392400 .

Shaw, T. A., and A. Voigt, 2016: What can moist thermodynamics tell us about circulation shifts in response to uniform warming? Geophys. Res. Lett., 43, 4566-4575.

Soden, B. J., I. M. Held, R. Colman, K. M. Shell, J. T. Kiehl, and C. A. Shields, 2008: Quantifying climate feedbacks using radiative kernels. J Climate, 21, 3504-3520.

Stone, P. H., and M.-S. Yao, 1990: Development of a two-dimensional zonally averaged statistical-dynamical model. Part III: The parameterization of the eddy fluxes of heat and moisture. Journal of Climate, 3 (7), 726-740.

Trossman, D. S., J. B. Palter, T. M. Merlis, Y. Huang, and Y. Xia, 2016: Large-scale ocean circulation-cloud interactions reduce the pace of transient climate change. Geophys. Res. Lett., 43, 3935-3943.

Zelinka, M. D., and D. L. Hartmann, 2012: Climate feedbacks and their implications for poleward energy flux changes in a warming climate. J. Climate, 25, 608-624. 